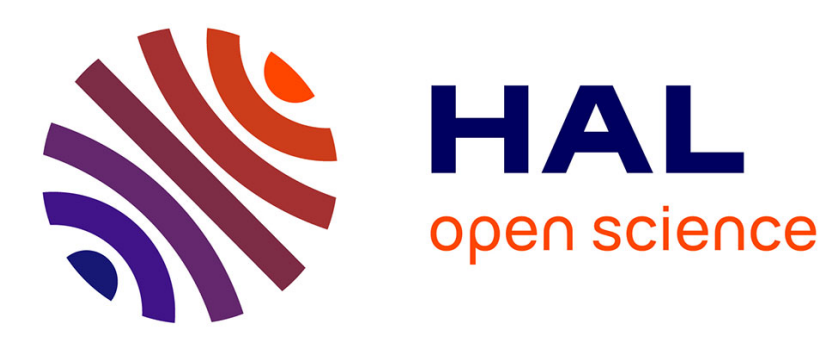

\title{
Phénomènes Diffusionnels et Recristallisation Accompagnant l'Assemblage de Superalliages Base Nickel par Compression à Chaud
}

Yves Bienvenu, F. Decla, J. Garcia

\section{- To cite this version:}

Yves Bienvenu, F. Decla, J. Garcia. Phénomènes Diffusionnels et Recristallisation Accompagnant l'Assemblage de Superalliages Base Nickel par Compression à Chaud. J. Phys. IV, 1995, 05 (C3), pp.C3-311-C3-316. 10.1051/jp4:1995332 . jpa-00253698

\section{HAL Id: jpa-00253698 https://hal.science/jpa-00253698}

Submitted on 1 Jan 1995

HAL is a multi-disciplinary open access archive for the deposit and dissemination of scientific research documents, whether they are published or not. The documents may come from teaching and research institutions in France or abroad, or from public or private research centers.
L'archive ouverte pluridisciplinaire HAL, est destinée au dépôt et à la diffusion de documents scientifiques de niveau recherche, publiés ou non, émanant des établissements d'enseignement et de recherche français ou étrangers, des laboratoires publics ou privés. 


\title{
Phénomènes Diffusionnels et Recristallisation Accompagnant l'Assemblage de Superalliages Base Nickel par Compression à Chaud
}

\author{
Y. Bienvenu, F. Decla et J.C. Garcia* \\ Centre des Matériaux, P.M. Fourt de l'Ecole des Mines de Paris, B.P. 87, 91003 Evry cedex, France \\ * TURBOMECA, Matériaux et Procédés, 40220 Tarnos
}

\begin{abstract}
Diffusion bonding is the prefered technique to join modern nickel based superalloys and produce hybrid aero-engine parts associating a disk type material (processed by powder metallurgy to resist low cycle fatigue at moderate temperature) and a large grain cast or directionally solidified material resisting creep. The microstructure in the vicinity of the joint, especially the $\gamma$ phase is affected by diffusion and mechanical properties are influenced by the transition from one structure to the other. The use of a ductile insert (nickel or nickelchromium) is a solution to perform a sound bond and to manage a transition in microstructures over distances reaching $100 \mu \mathrm{m}$, but diffusion phenomena are amplified. Recristallisation related aspects are discussed.
\end{abstract}

\section{INTRODUCTION}

L'évolution des conditions de fonctionnement ( $T$, température, $\sigma$, contrainte) des disques chauds des turbines aéronautiques amène à étudier la substitution du concept de monomatériau et monostructure par celui de bimatériau (assemblage fixe aube-disque) tout en conservant les superalliages base $\mathrm{Ni}$. En effet, au delà de $700^{\circ} \mathrm{C}$ (température atteinte dans les aubes), le fluage devient prépondérant pour cette classe d'alliage et conduit à choisir une structure à grains orientés voire monocristalline pour $1050^{\circ} \mathrm{C}$ et plus. Les moyeux de disques doivent faire face à des températures plus modestes $\left(450^{\circ} \mathrm{C}\right)$ mais à des contraintes élevées et cycliques (pouvant dépasser $1000 \mathrm{MPa}$ ), d'où le choix local d'une structure à grain fin à limite d'élasticité et limite d'endurance élevées.

Si le choix de ces matériaux et de ces structures ne pose pas de problème, leur assemblage reste une opération difficile. En effet, la structure de ces alliages renforcée par précipitation de $\gamma$ ne permet pas l'utilisation d'une technique classique de soudage par fusion (Tig, bombardement électronique, etc...). De plus, les fixations mécaniques actuelles en pieds de sapin des aubes sur les disques montrent leurs limites. Ceci nous conduit à envisager une liaison métallurgique par soudage diffusion en compression à chaud isostatique ou uniaxiale (C.I.C. ou C.U.C.) [1-4]. Le soudage par friction est aussi envisageable mais du fait du niveau de déformation, son application est limitée aux matériaux pour disques [1]. Le brasage-diffusion est la technologie d'assemblage des superalliages la plus simple à mettre en oeuvre mais la présence de borures, siliciures, d'intermétalliques en plus de porosités à l'interface est un désavantage .

Le soudage par diffusion permet d'assembler deux alliages de structure différente avec une déformation plastique limitée. Cependant, les différences de composition des alliages ainsi que les températures élevées de traitement peuvent entraîner des phénomènes de diffusion et de recristallisation au niveau du joint $[2,3,5]$. La recristallisation est à éviter ou à limiter pour les aubes monocristallines. 


\section{MATERIAUX ET PROCEDES}

Le couple de superalliages retenu pour cette étude est Astroloy/DS247. L'Astroloy (NK17CDAT), élaboré par compaction isostatique à chaud de poudres préalliées est un superalliage de reférence dans la classe des matériaux destinés aux disques de turbine : c'est un alliage à grain fin (10 à $30 \mu \mathrm{m}$ suivant les traitements thermiques) avec de bonnes propriétés en fatigue oligocyclique jusqu'à $650^{\circ} \mathrm{C}$ et dont la fraction volumique de $\gamma^{\prime}$ est ${ }^{~}$ de $45 \%$.

Le DS247 (NKW9CATaHf) est un alliage à gros grains pour aubes obtenu par solidification dirigée. Le but de cette technologie est de supprimer les joints de grains orthogonaux à l'axe de sollicitation afin d'augmenter la résistance au fluage. Des superalliages monocristallins sont dérivés de cette composition.

Tableau 1. Composition et solvus $\gamma^{\prime}$ des deux superalliages utilisés (masse.\%, $\mathrm{Ni}=$ reste)

\begin{tabular}{|c|c|c|c|c|c|c|c|c|}
\cline { 2 - 8 } \multicolumn{1}{c|}{} & $\mathrm{Co}$ & $\mathrm{Cr}$ & $\mathrm{Al}$ & $\mathrm{Ti}$ & $\mathrm{Mo}$ & $\mathrm{W}, \mathrm{Ta}, \mathrm{Hf}$ & $\mathrm{C}$ & $\begin{array}{c}\gamma^{\prime} \text { Solvus } \\
\mathrm{C}\end{array}$ \\
\hline Astroloy & 16.9 & 14.8 & 3.87 & 3.45 & 5.1 & - & 0.015 & 1135 \\
\hline DS 247 & 10 & 8 & 5.5 & 1 & 0.6 & $\begin{array}{c}10 . \mathrm{W} \\
3.0 \mathrm{Ta} \\
1.5 \mathrm{Hf}\end{array}$ & 0.06 & 1235 \\
\hline
\end{tabular}

La température de solvus $\gamma^{\prime}$ est la température caractéristique au dessus de laquelle les précipités $\gamma^{\prime}$, cohérents avec la matrice $\gamma$ de structure C.F.C., se dissolvent, laissant ainsi le grain libre de grossir.

Pour l'étude que nous présentons, la liaison est effectuée en compaction uniaxiale à chaud (C.U.C.) avec les conditions suivantes :

- contrainte uniaxiale de $10 \mathrm{MPa}$, inférieure à la contrainte d'écoulement de l'Astroloy,

- Palier de $3 \mathrm{~h}$ à $1180^{\circ} \mathrm{C}$, température intermédiaire entre les deux solvus,

- Enceinte sous vide secondaire, pour limiter les phénomènes d'oxydation.

Cette technique d'assemblage à chaud est plus souple et précise en température que la C.I.C. et l'expérience a prouvé que la différence au niveau des contraintes appliquées par rapport à la C.I.C.. est sans grande répercussion sur la microstructure. Les deux matériaux sont sous forme de pions cylindriques de $\varnothing 10 \mathrm{~mm}$ ou $8 \mathrm{~mm}$. Le mode de préparation de la surface des pions avant assemblage et le mode de réalisation d'un insert (feuillard intercalé ou dépôts plasma) ne seront pas discutés en détail dans cette présentation des aspects liés à la recristallisation.

\section{RESULTATS ET DISCUSSION}

\subsection{Intérêt de $\mathrm{Ni}-\mathrm{Cr}(80 \%-20 \%)$ comme insert}

Les études précédentes ont montré la nécessité de la présence d'un insert ductile entre les deux éléments à assembler [3]. En effet, la faible déformation plastique des superalliages à la température de compaction ne peut résorber les aspérités en certains points de l'interface initial. L'insert, non durci par $\gamma$ ou par W, Mo, Ta, jouera le rôle de régulateur des imperfections de surface grâce à sa ductilité [3]. Toutefois, l'apport d'un matériau supplémentaire entraîne d'importantes perturbations de la microstructure au niveau du joint. Il convient donc d'optimiser sa composition et son mode d'apport pour limiter les défauts de sa structure et faciliter la transition microstructurale.

Une étude antérieure [3] a montré que l'utilisation d'un insert de nickel pur permet de garantir l'absence de porosité mais entraîne la formation de gros précipités $\gamma^{\prime}(>10 \mu \mathrm{m})$ à l'interface responsables d'un mauvais comportement en fluage, les caractéristiques en traction et en fatigue $\left(650^{\circ} \mathrm{C}\right.$ et $\left.750^{\circ} \mathrm{C}\right)$ étant 
satisfaisantes. Une variation de la composition chimique de l'insert devrait ici permettre d'éliminer ce défaut. L'idée de base est de décaler les compositions dans l'insert modifié par diffusion vers la phase $\gamma$ en rajoutant un élément $\gamma$-gène comme le chrome. Sur les diagrammes ternaires $(\mathrm{Al}, \mathrm{Cr}, \mathrm{Ni})$ de la figure 1 , nous avons placé deux points représentatifs de l'Astroloy (45\% de $\left.\gamma^{\prime}\right)$ et le DS247 (70\% de $\left.\gamma^{\prime}\right)$. Ceux-ci étant constitutés d'une douzaine d'éléments, il ne s'agit ici que d'une représentation schématique. Nous pouvons aussi tracer des chemins de diffusion dans le cas où l'on utilise un insert de nickel ou un insert d'un alliage $\mathrm{Ni}(80 \%) \mathrm{Cr}(20 \%)$ à partir des profils de composition (analyse à la microsonde de Castaing sur sections polies d'assemblages effectués à $1180-1200^{\circ} \mathrm{C}$ ). Le chemin de diffusion dans le cas de l'insert nickel s'explique par le fait que l'aluminium diffuse beaucoup plus vite que le chrome. On peut ainsi voir qu'en utilisant un insert de nickel, on se rapproche du domaine $\gamma^{\prime}$, alors qu'avec un insert nickel-chrome, on s'en éloigne, d'où une plus grande régularité dans l'évolution de la taille et la fraction volumique des précipités $\gamma^{\prime}$ en principe. L'épaisseur de l'insert a été variée de 10 à $40 \mu \mathrm{m}$ dans notre étude, l'optimum, au vu des études microstructurales et microanalytiques, se situant à $20 \mu \mathrm{m}$ (obtention d'une précipitation gamma prime dans l'ensemble de l'insert en 3 heures de diffusion).

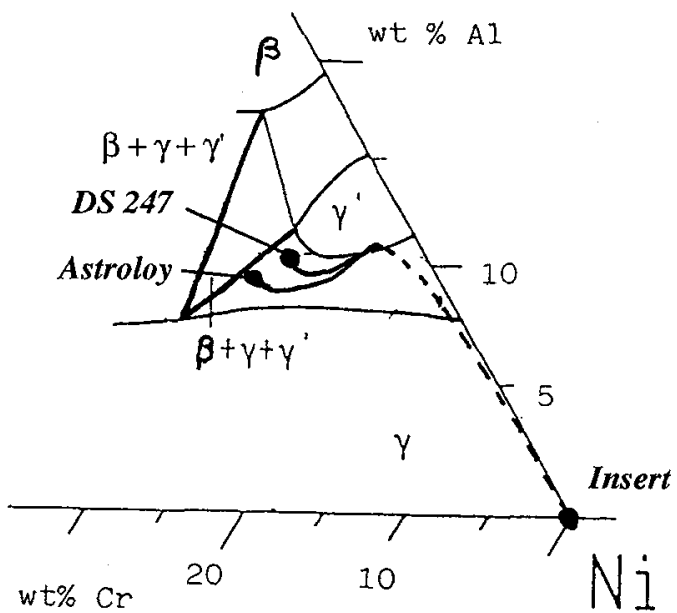

(a)

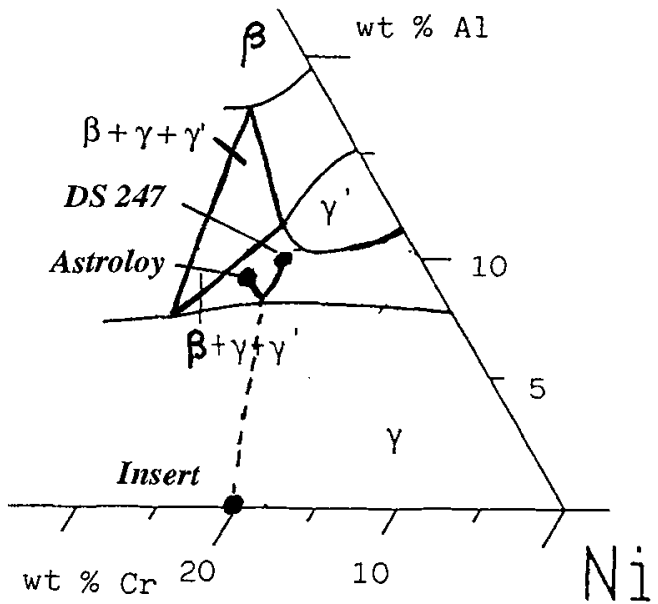

(b)

Figure 1 Report dans le diagtamme ternaire (section isotherme à $1150^{\circ} \mathrm{C}$ ) [7] des chemins de diffusion Astroloy /insert / DS247 dans ce cas d'un insert en Ni pur (a) et en Ni-20Cr (b) (représentation schématique).

\subsection{Migration de l'interface monophasé/biphasé à la température d'assemblage}

L'assemblage étant réalisé à une température intermédiaire entre les deux températures de solvus, la phase $\gamma$ est complètement remise en solution dans l'Astroloy mais pas dans le DS247. On a donc, pendant le palier en température, une partie monophasée (Astroloy + joint) et une partie biphasée (DS247). Les gradients de concentration déclenchent des phénomènes de diffusion importants. Ainsi, l'insert qui ne contient que du nickel et du chrome va jouer le rôle de puits de diffusion, pour l'aluminium et le titane, tous deux $\gamma^{\prime}$-gènes, ce qui entraîne, dans le DS247 au contact de l'insert, une rupture de l'équilibre $\gamma / \gamma^{\prime}$ et localement un abaissement de la température de solvus (figure 2) sous la température de soudage $\left(1180^{\circ} \mathrm{C}\right)$. La remise en solution des $\gamma$ du DS247 proches du joint se traduira par une avancée de la frontière monophasé/ biphasé (figure $3 \mathrm{~b}$ ) à $1180^{\circ} \mathrm{C}$ et une différence de morphologie de précipitation après refroidissement lent dans le four presse qui donne à la microstructure de l'assemblage cette allure dissymétrique (fïgure 3-c). 


\section{Insert $\mathrm{Ni}-\mathrm{Cr} 20 \mu \mathrm{m}$}

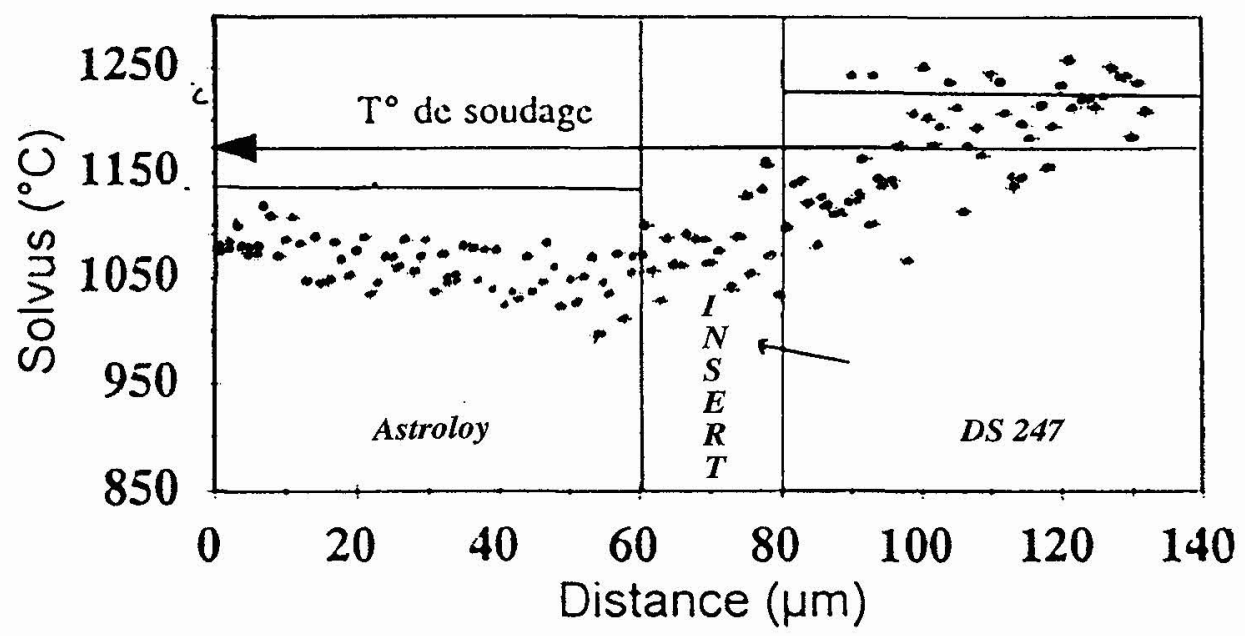

Figure 2 Evolution de la température de solvus calculée à partir des compositions locales (profil microsonde.

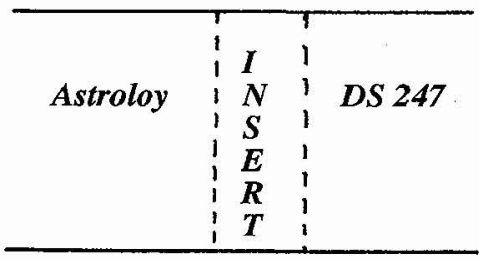

a) Situation initiale

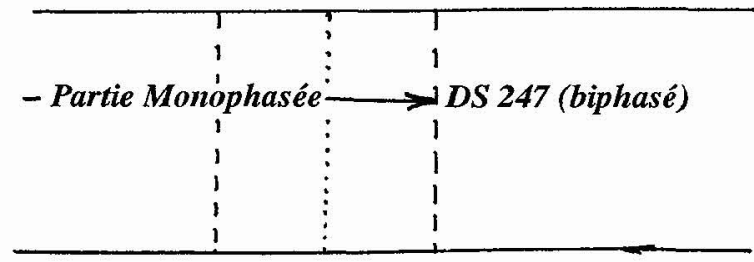

b) après diffusion

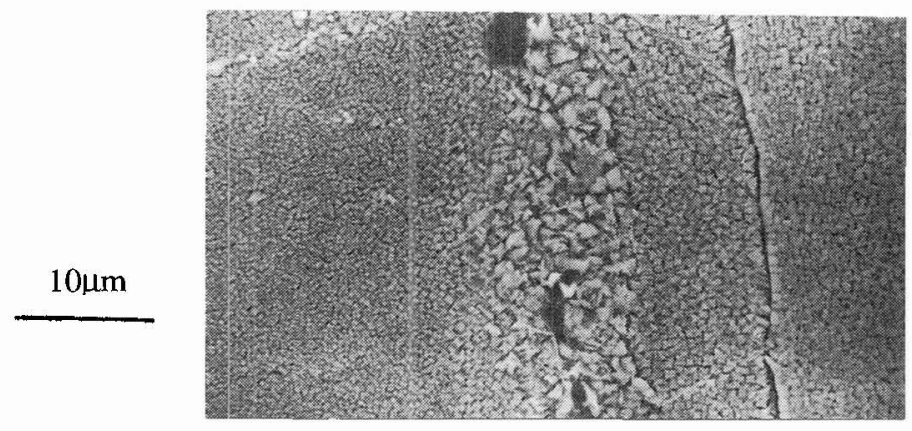

c) micrographie de b

Figure 3 Evolution de la microstructure au niveau du joint après pressage $3 \mathrm{~h}$ à $1180^{\circ} \mathrm{C}$.

Cette nouvelle frontière (fígure 3) ne marque pas forcément un nouveau joint de grain. En effet, la précipitation des $\gamma^{\prime}$ est intra-granulaire et cohérente avec la matrice et il ne semble pas, à observer l'orientation des précipités $\gamma$ dans ce liseré ex-DS247, que l'orientation de la phase $\gamma$ ait varié mais ce 
point reste à préciser. Aucune recristallisation généralisée de l'alliage DS247 n'a été observée dans tous les cas. Une étude antérieure sur l'assemblage DS247/DS247 à $1250^{\circ} \mathrm{C}$ avait cependant montré qu'une recristallisation pouvait intervenir sur $20 \mu \mathrm{m}$ environ dans le cas où la finition de surface du DS247 laisse des contraintes résiduelles importantes (rectilication on grenaillage) avant assemblage [6].

L'évolution de la précipitation $\gamma^{\prime}$ au travers du joint avec $\mathrm{Ni}-20 \mathrm{Cr}$ est plus régulière qu'avec l'insert $\mathrm{Ni}$ [3]. La taille des précipités n'excède pas $5 \mu \mathrm{m}$. Des études pour différents couples de superalliages assemblés vers $1100^{\circ} \mathrm{C}[2,3]$ et différents temps de palier ont montré que la cinétique d'avancée de la structure monophasée est parabolique avec une constante de $10^{-10} \mathrm{~cm}^{2} / \mathrm{s}$ environ [6].

\subsection{Apparition de $\gamma$ cellulaires}

Il apparaît parfois et localement le long du joint DS247/insert des liserés ou des "boutonnières" de "gros" $\gamma$ de formes cellulaires dans le DS247. A.W. Funkenbusch [5] explique ce phénomène de coalescence destiné à diminuer les énergies d'interface par un mécanisme de dissolution/reprécipitation. La distance affectée (moins de $15 \mu \mathrm{m}$ ) de cette précipitation discontinue va donc être conditionnée par la vitesse du front de dissolution, c'est-à-dire par la diffusion aux joints de grains et par une force motrice pour la dissolution / reprécipitation et la durée de l'assemblage .En plus de la réduction des tensions interfaciales, les forces motrices sont les contraintes résiduelles et la densité de dislocations élevée. La figure 4 présente l'aspect d'une liaison pour laquelle les deux surfaces en contact avaient été préalablement grenaillées avec des billes de nickel, circonstance permettant la précipitation cellulaire généralisée le long du joint.

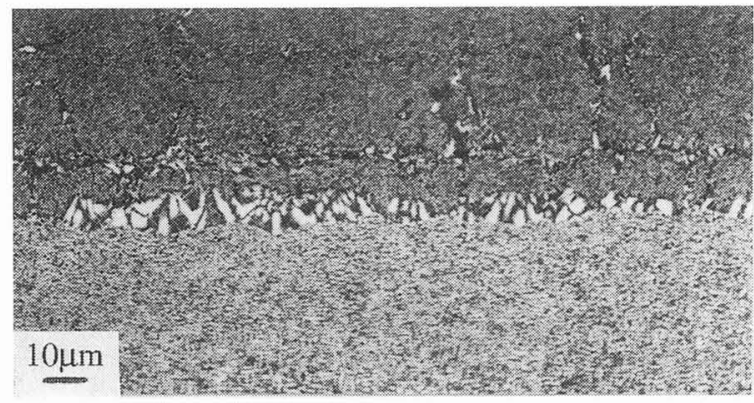

\section{Insert \\ DS 247}

Figure 4 Exemple de précipitation cellulaire de $\gamma^{\prime}$ à l'interface DS247 / insert (généralisée à cause de la pratique d'un grenaillage avant soudage).

\subsection{Répercussions sur les caractéristiques mécaniques des assemblages (étude préliminaire)}

Des essais de traction à $20^{\circ} \mathrm{C}$ ont montré que la liaison mécanique entre les deux matériaux était bien réalisée. En effet, on constate une déformation plastique non négligeable (de l'ordre de $2 \%$ ) et un niveau de contrainte à rupture élevée, inférieur de $10 \%$ environ à la contrainte à rupture du materiau le plus faible. La rupture n'est pas située à l'interface initiale mais dans une zone proche de celui-ci.

Il reste cependant à compléter la caractérisation mécanique (en particulier fluage). Le grossissement des grains de l'Astroloy lors de l'assemblage à $1180^{\circ} \mathrm{C}$ devrait entraîner une baisse de la limite d'élasticité et de la contrainte à la rupture ainsi qu'une augmentation de la ductilité. Des gains importants peuvent être obtenus sur les propriétés mécaniques des assemblages en optimisant les traitements thermiques et en limitant la formation de carbures et oxydes interfaciaux. 


\section{CONCLUSION}

Les phénomènes qui contrôlent la précipitation de la phase $\gamma$ sont de nature diffusionnelle. Un insert de nickel $(80 \%)$-chrome $(20 \%)$ de $20 \mu \mathrm{m}$ d'épaisseur donne de bons résultats au niveau de la microstructure. Il est suffisamment ductile pour éviter la présence de porosités interfaciales et permet d'éviter la formation de gros précipités de $\gamma$.

Il reste à effectuer une caractérisation mécanique complète et à étudier de plus près la recristallisation de l'insert qui semble "colonisé" par l'Astroloy. La recristallisation généralisée de l'alliage DS247 n'est pas observée même dans le cas d'un état de surface grossier. Les seuls phénomènes de type recrisallisation concernent la précipitation cellulaire de $\gamma^{\prime}$ et sont limités à une fraction de la profondeur caractéristique de diffusion. La diffusion contrôle les évolutions microstructurales et des bases de modélisation sont proposées. Une transition entre les deux microstructures peut être obtenue sur $100 \mu \mathrm{m}$ environ par l'épaisseur de l'insert et des distances caractéristiques de diffusion.

\section{REFERENCES}

[1] R.C. Wing and M. Newham, Dual alloy turbine blisk, Diffusion Bonding, Roger Pearce ed., S.I.S., Cranfield Inst. of Tech., Cranfield, UK, 1987, p 159

[2] Y. Bienvenu, B. Decaudin, T.J. Massart and F. Schwartz, Metallurgical aspects of solid joining of nickel base superalloys, in $6^{\text {th }}$ International Conference, High Technology Joining, Strafford, 3-5 Sept. 1991, B.A.B.S., Abingdon, U.K. ed., (1991), p 7.

[3] Y. Bienvenu, M.L. Dupont, F. Schwartz et J.C. Garcia, Assemblage par soudage diffusion de deux superalliages. Aspects diffusionnels et microstructuraux au niveau du joint, Journal de Physique IV, Colloque C3, Vol.4, 1994, p 117.

[4] H. Takigawa et al., Process development for low cost high strengh P.M. Ni-base superalloy turbine dics, Metal. Powd. Rep. 44, 9, 1986, p 602.

[5] A.W. Funkenbusch, Discontinuous $\gamma^{\prime}$ coarsening in Ni-Al-Mo base superalloy, Metallurgical Transactions, vol. 14.A, July 1983, pp 1283-1292.

[6] T.J. Massart, A. Morisson, J.L. Koutny et Y. Bienvenu, Soudage par diffusion de superalliages à base nickel : Répercussions microstructurales, Recueil des 4e Journées Nationales du Soudage; Institut de Soudure et Société des Ingénieurs Soudeurs, 14-16 mars 1989, Paris.

[7] Ternary Alloys, a compendium of phase diagrams, ed. by G. Petzow, WCH ed. Weinheim, New York, 1991.

Remerciements : Les auteurs tiennent à remercier G. Frot (microanalyse) et $F . N^{t} G u y e n$ (analyse d'images) du Centre des Matériaux, G. Marty et M. Soucail de l'ONERA pour calculs et discussions au sujet de la stabilité de la phase $\gamma$. 